\title{
Examining the use of geriatric assessment teams and comprehensive geriatric assessment in emergency departments
}

\author{
By Mathieu Figeys, BScN, RN, Naweed Ahmed, MD, and Jennifer Evans, BScN, RN
}

\section{Introduction}

With the rapidly growing population of adults over the age of sixty-five, emergency departments are seeing a markedly increased number of geriatric patients seeking acute care. Although many geriatric patients are admitted to inpatient wards, often times they are discharged back into their community setting. It is important to note that emergency department staff are often not geriatric specialists. Further, the current emergency care model of rapid 'door-to-disposition' often overlooks the complexity of geriatric patients, who have a higher prevalence of comorbidities.

To address this gap in healthcare, and to better meet the needs of geriatric patients presenting to the emergency department, geriatric assessment teams are becoming increasingly prevalent in acute care practice. The objectives of this paper are three-fold, and will be addressed from a social epidemiological standpoint; firstly, to explore trends in geriatric presentations to the emergency department, secondly, to discuss healthcare disparities in acute care services for geriatric patients, and lastly, to discuss the impact of geriatric assessment teams on the patient and healthcare system. Furthermore, stemming from the discussion, potential policy implications within the acute healthcare system will be highlighted.

\section{Background}

lobally, the population of the aged adult over the age of sixty-five will grow by one-billion people by the year 2050 (Asomaning \& Loftus, 2014). Currently, patients over the age of sixty-five make up $11 \%$ of the total population of patients presenting to Canadian emergency departments (Baumbusch \& Shaw, 2011). With the increasing aging population, a rise of geriatric patients presenting to emergency departments will follow. Geriatric patients are also significantly more likely to be admitted to formal inpatient services, and have a higher rate of adverse health outcomes post-discharge from an emergency department (Keyes, Singal, Kropf, \& Fisk, 2014). Furthermore, geriatric patients in emergency departments are more likely to have diagnostic imaging and longer lengths of stay (LaMantia et al., 2010), and are more likely to require costlier care compared to their younger counterparts (Hwang \& Morrison, 2007). Considering these factors, it is important to realize that the Canadian healthcare system was designed to treat acute illness instead of chronic illness, and designed for a younger population (Macintosh, Rajakulendran, Khayat, \& Wise, 2016). Although gaps in healthcare are being addressed through the current healthcare transformation, geriatric patients remain at a disadvantage.

\section{Methods}

A review of published literature was performed using the Ovid Medline and CINAHL databases (refer to Appendix A for the search criteria). Search results were compiled into Mendeley [Mendeley Ltd, London, United Kingdom], and duplicate results were removed. A minimum age restriction was placed, at 65 years old, to capture the complexity of health for all aged adults and to exclude younger adults. Articles in English were reviewed, without restriction on country of publication. Articles published before 2010 were excluded and no restriction on patient sex was employed. Furthermore, additional supporting literature was found using Google scholar, without publication date limitations. Instead of conducting a meta-synthesis or meta-analysis, this paper is focused on promoting awareness of geriatric presentations in emergency departments, highlighting the role of geriatric assessment teams, and discussing policy implications in the Canadian acute care system.

\section{Geriatric considerations in emergency care}

Geriatric patients often have complex biological and psychosocial health presentations that amalgamate in age from the individual's life course, such as biological, environmental, and social barriers (Sadana, Blas, Budhwani, Koller, \& Paraje, 2016). Due to this, atypical presentations are more prevalent in geriatric patients. For example, atypical presentations of acute coronary syndromes in geriatric patients are more prevalent compared to their younger counterparts (Salvi, Morichi, Grilli, Giorgi, De Tommaso, \& DessìFulgheri, 2007).

Frailty can be defined as "...the inability to withstand illness without loss of function or a loss of functional homeostasis. It is a complex interplay of multi-morbidity and aging physiology” (Ellis, Marshall, \& Ritchie, 2014, pp. 2034). Those with frailty are predisposed to falls, delirium, elimination issues, caregiver burnout, and immobility (Ellis et al., 2014). The complexity of frailty is often minimally assessed by emergency 
practitioners due to time constraints, nonspecific syndrome presentations, and by being vague during history assessment (Ellis et al., 2014).

As Salvi et al. suggest, comorbidity in older adults only complicates differential diagnoses and disposition (2007). Furthermore, delirium is commonly seen in geriatric patients in the emergency department, however it is widely under-diagnosed (Salvi et al., 2007). Progressive cognitive impairment (such as dementia), is seen in $15-40 \%$ of aged adults presenting to the emergency room, however, like delirium, it is under-recognized resulting in gaps in care (Salvi et al., 2007). To add to the complexity of care, geriatric patients are more frequently predisposed to polypharmacy, and adverse drug reactions due to age related pharmacokinetics and pharmacodynamics, which is rarely assessed by emergency practitioners (Salvi et al., 2007; Samaras, Chevalley, Samaras, \& Gold, 2010). Although functional decline is well documented as a predictor of early mortality, the majority of emergency practitioners fail to assess functional status despite the fact that only $22 \%$ of elderly patients presenting to the emergency department are independent with their activities of daily living (Salvi et al., 2007; Extermann et al., 2005).

Moving beyond geriatric health factors, emergency departments are also failing to address geriatric care in regards to the environment where the care is being provided. Hwang \& Morrison (2007) suggest that the current design of emergency departments is intended for efficiency; they lack privacy, comfort, promote fall hazards, have poor lighting, and are loud. Hence it is also important to address how care space influences geriatric patients. To promote geriatric care in the emergency department, increased resources need to be made available for assessment, interventions, and to promote an elder friendly environment.

\section{Geriatric assessment in the emergency department}

There is an evident need for geriatric specialists within the emergency department. Some emergency departments are dedicating geriatric-specific beds to allow geriatric teams to assess patients. Further, some regions are now exploring the creation of geriatric emergency departments specifically designed for older adults (Ellis et al., 2014). Recent literature demonstrates that geriatric friendly emergency departments reduce admission rate, though length of stay and return visits remained unchanged (Keyes et al., 2014).

One strategy that shows promising results is the creation of interdisciplinary geriatric assessment teams who are involved in the care of elderly adults, and the rise of the comprehensive geriatric assessment (CGA). The utilization of CGA has been demonstrated to reduce early mortality, and increase self-functioning (Leclerc et al., 2013). The CGA is an assessment tool developed for a multidisciplinary approach that addresses: cognitive evaluation, mood evaluation, comorbidity evaluation, polypharmacy review, falls assessment, functional status, social supports, and nutrition (Graf, Zekry, Giannelli, Michel, \& Chevalley, 2011).

Leclerc et al., 2013, discuss the role of inpatient geriatric assessment in Quebec, where assessment teams are composed of medical physicians, nurses, physical therapists, occupational therapists, social workers, dietitians, and pharmacists. Geriatric assessment requires multiple assessment points, hence an adequate length of stay (approximately three weeks); efficient care management, and correct admission location are key characteristics of a detailed geriatric assessment (Leclerc et al., 2013). Furthermore, the CGA is a timely process, which often times emergency caregivers do not have adequate time to perform (Graf et al., 2011). Thus, the question arises: how can the complexity of geriatric assessment be performed within the short stay in the acute healthcare setting?

Graf et al., (2011), propose a model where the CGA is not assessed in every geriatric patient. Some geriatric patients live independently with a high level of functional and cognitive status. Their study found that other validated geriatric screening tools, which can be performed quickly by emergency physicians, include the Identification of Seniors at Risk (ISAR), and the Triage Risk Screening Tool (TRST). These abbreviated tests are conducted to identify high-risk patients, whom the CGA should be performed on (Graf et al., 2011).

Geriatric assessments teams are becoming increasingly prevalent in emergency departments to attempt to address the gaps between the healthcare system and elderly patients. Drawing from a UK study by Conroy et al., (2014), the introduction of comprehensive geriatric assessment in the emergency department with dedicated geriatric beds reduced inpatient admissions, and decreased re-presentation to the emergency department. It is also suggested that CGA use increased the efficiency of care for younger adults, as the emergency practitioners had more allocated time as the geriatric team cares for medically complex aged adults (Conroy et al., 2014). Caplan, Williams, Daly, \& Abraham (2004) found that outpatient CGA referral conducted by a hospital-based interdisciplinary outreach team resulted in a lower hospital admission rate thirty-day post emergency department visit, and resulted in a ten percent reduction of emergency visits at 18 months. Hence, the researchers promote an outpatient CGA referral to adults over the age of 75 after an emergency department visit (Caplan et al., 2004).

Lastly, geriatric emergency nurses (GENs) are also becoming prevalent in practice. Baumbusch and Shaw (2011) examined the role of the geriatric emergency role in a hospital in Vancouver. The researchers found that GENs often act as case managers within the emergency department, specialize in geriatric assessment and geriatric care planning, and creating outpatient dispositions, follow-ups, and community referrals (Baumbusch \& Shaw, 2011). Flynn and Ryan, 2012, identify five categories of GEN interventions: geriatric emergency management, targeted assessment, screening and referring, consultation and creating recommendations, and following-up with patients. Their specialist knowledge in geriatric training and emergency medicine provide the continuity of care often needed in complex geriatric patients.

\section{Current interventions and future directions}

Sunnybrook Hospital first implemented a GEN in 2002 with positive feedback, resulting in the creation of further positions. Between April 2005-2006, almost 3,000 seniors were seen by eight GENs, of which nearly half were identified as being at 
risk (Flynn \& Ryan, 2012). Furthermore, it reduced the average length of stay to ten hospital days (from 17.5 days), and reduced post-ED hospital admission rate by four percent (Flynn \& Ryan, 2012). As of 2012 statistics, 102 GENs staff 53 emergency departments in Ontario. In addition, Ontario has emergency-based mobile nurses to provide emergency nursing care in the home-setting, instead of transporting the patient to an emergency department. Between 2011-2012, nine emergency mobile nurses existed, which saw over 10,000 patient encounters, which significantly reduced the number of transports to emergency departments (Flynn \& Ryan, 2012). Similarly, in other provinces (such as Alberta) community paramedics are also beginning to provide at-home services instead of transporting patients to an emergency facility.

There is an increasing recognition from emergency staff regarding knowledge gaps that are necessary to fill to prepare for the rapidly aging senior population. Flynn and Ryan (2012) report that one percent of emergency practitioners have geriatric expertise, and that there is a lack of clinical schooling in geriatrics. There is an obvious need to educate frontline emergency staff regarding geriatric health and aging. Furthermore, there is a significant potential for cost-saving measures by implementing specialist geriatric assessment teams in emergency departments as previously discussed. Using 2013-2014 Alberta figure estimates from the Institute of Health Economics, the operational cost for an ambulance transport is $\$ 760.48$ per event, the average cost per day in a medical ward is nearly $\$ 1,000$, and the average cost per day in a critical care bed is $\$ 4,114.00$ (Institute of Health Economics, n.d.). Extrapolating these results to other provinces, the utilization of geriatric assessment teams and community-based interventions could save each provincial/territorial healthcare system millions of dollars annually.

Additional research needs to be conducted in regards to geriatric emergency medicine, such as screening instruments and tools, the role of emergency nursing in geriatric patients, the creation of geriatric emergency departments, frailty in the emergency context and geriatric assessment teams. The rise of telemedicine and geriatric emergency medicine should further be explored, especially in rural settings. Adequate resources for these teams are required, as patient caseloads will only increase as the population ages. Community resources need to increase awareness, and be made available and accessible to geriatric assessment teams, patients, and their caregivers. Organization of care needs to be re-addressed to remove barriers for our senior adults. The creation of dedicated geriatric assessment beds in Canadian emergency departments is a future area of exploration to prepare for the rapid influx of aged adults seeking emergency care. The creation or renovation of emergency departments should also reflect a safe environment for geriatric patients.

\section{Conclusion}

Geriatric patients often present to emergency departments with complex factors influencing their individual health. The current outdated design of emergency healthcare place geriatric patients at a disadvantage, and can be a potential barrier to healthcare.
The population is aging, and efforts need to be invested to meet care demands for aged adults, and prepare for the future increase in geriatric care in emergency settings. Trends in geriatric populations have been discussed, such as frailty, multimorbidity, polypharmacy, and functional decline. Healthcare gaps have been briefly identified, and potential solutions, such as geriatric assessment teams in emergency care settings, have been discussed as a method to fill in the existing gap between geriatric and emergency medicine. We must continue to reform the healthcare system for geriatric clients, adapt as new technologies and methodologies arise, and prepare for our future.

Beeping, chatter, clanging, banging and yelling are only a few distinct sounds piercing one's ear in the ER. For a senior, it can be quite different than the comforts of home-noting the whistle of tea kettle brewing and the TV channel set to their routine show. Working in both the community for several years with many seniors directly in their homes as a case manager (CM), and now working in the ER as a transition coordinator (TC) since 2014, the environments can be most of the time polar opposite. As a coordinator, I help bridge the gap between hospital and home as a home care liaison, advocate, and voice for continuing care clients, mostly seniors over the age of 65 . In Edmonton, there have been positions created trying to ease the pain, by providing continuity of care, being able to see both patient in the ER and then at home. They are system case managers (SCM) and I have the privilege of working closely with them to help complex continuing care patients transition from hospital to home. If they are medically stable and able to return home safely, it is cheaper to keep someone at home than to be admitted into an acute care bed or be waiting to be admitted from an ER bed. By having multiple health care professionals involved in care planning, with geriatric knowledge, it allows set up for quicker transitions home, smoother communication, appropriate service utilization, and keeps patients out of the hospital who don't need to be, and risks related to hospitalization are avoided. The continuity of care is of utmost importance and for a senior to see a familiar face really helps with rapport and care. I have had the privilege of also being able to access a geriatric NP and geriatrician to complete assessments in the ER, and have further follow up in the community if needed from the same geriatric professionals. As a TC, being able to triage and assess seniors' care needs, while referring to community supports to keep patients out of hospital, relates closely to that of a geriatric ER nurse (GEN), and according to Sunnybrook Hospital in Toronto: there is research suggesting that the use of GENs decreased admission and re-presentations to the emergency room (Flynn \& Ryan, 2012). I would hope in the future these approaches are utilized throughout ERs across the country if they haven't started already, to assist with the flow of the hustle and bustle of the emergency department.

\author{
-Jennifer Evans, BScN, RN \\ Transition Coordinator \\ Royal Alexandra Hospital, Edmonton, Alberta
}


Asomaning, N., \& Loftus, C. (2014). Identification of seniors at risk (ISAR) screening tool in the emergency department: Implementation using the plan-do-study-act model and validation results. Journal of Emergency Nursing: JEN: Official Publication of the Emergency Department Nurses Association, 40(4), 357-364.e1. doi:10.1016/j.jen.2013.08.014

Baumbusch, J., \& Shaw, M. (2011). Geriatric emergency nurses: Addressing the needs of an aging population. Journal of Emergency Nursing, 37(4), 321-327. doi:10.1016/j.jen.2010.04.013

Caplan, G.A., Williams, A.J., Daly, B., \& Abraham, K. (2004). A randomized, controlled trial of comprehensive geriatric assessment and multidisciplinary intervention after discharge of elderly from the emergency Department-The DEED II study. Journal of the American Geriatrics Society, 52(9), 1417-1423. doi:10.1111/j.1532-5415.2004.52401.x

Conroy, S.P., Ansari, K., Williams, M., Laithwaite, E., Teasdale, B., Dawson, J., ... Banerjee, J. (2014). A controlled evaluation of comprehensive geriatric assessment in the emergency department: The 'emergency frailty unit'. Age and Ageing, 43(1), 109-114. doi:10.1093/ageing/aft087

Ellis, G., Marshall, T., \& Ritchie, C. (2014). Comprehensive geriatric assessment in the emergency department. Clinical Interventions in Aging, 9, 2033-2043. doi:10.2147/CIA.S29662

Extermann, M., Aapro, M., Bernabei, R., Cohen, H.J., Droz, J., Lichtman, S., ... Task Force on CGA of the International Society of Geriatric Oncology. (2005). Use of comprehensive geriatric assessment in older cancer patients: Recommendations from the task force on CGA of the international society of geriatric oncology (SIOG). Critical Reviews in oncology/hematology, 55(3), 241.

Flynn, D., \& Ryan, D. (2012). Older adults in the emergency department: A senior friendly approach to care. Speech presented at Manitoba Gerontological Nursing Association Education Day, Winnipeg. Retrieved July 16, 2017, from http://gem.rgp.toronto. on.ca/files/Materials\%20for\%20the\%20October\%202012\%20 Manitoba\%20Geriatric\%20Nursing\%20Association\%20 Education\%20Day\%20for\%20web_0.pdf

Graf, C.E., Zekry, D., Giannelli, S., Michel, J., \& Chevalley, T. (2011). Efficiency and applicability of comprehensive geriatric assessment in the emergency department: A systematic review. Aging Clinical and Experimental Research, 23(4), 244-254. doi:10.1007/ BF03337751

Hwang, U., \& Morrison, R.S. (2007). Models of geriatric care, quality improvement, and program dissemination: The geriatric emergency department. Journal of the American Geriatrics Society, 55(11), 1873. doi:10.1111/j.1532-5415.2007.01400.x

Institute of Health Economics. (n.d.). Alberta costing approach. Retrieved from http://obrieniph.ucalgary.ca/files/iph/table-3. pdf

Keyes, D.C., Singal, B., Kropf, C.W., \& Fisk, A. (2014). Impact of a new senior emergency department on emergency department recidivism, rate of hospital admission, and hospital length of stay. Annals of Emergency Medicine, 63(5), 517-524. doi:10.1016/j. annemergmed.2013.10.033

LaMantia, M.A., Platts-Mills, T.F., Biese, K., Khandelwal, C., Forbach, C., Cairns, C.B., ... Kizer, J.S. (2010). Predicting hospital admission and returns to the emergency department for elderly patients. Academic Emergency Medicine, 17(3), 252-259. doi:10.1111/j.1553-2712.2009.00675.x

Leclerc, B., Presse, N., Bolduc, A., Dutilleul, A., Couturier, Y., \& Kergoat, M. (2013). Interprofessional meetings in geriatric assessment units: A matter of care organization. Journal of Interprofessional Care, 27(6), 515-519. doi:10.3109/13561820.2013.807778

Macintosh, E., Rajakulendran, N., Khayat, Z., \& Wise, A. (2016). Transforming health: Shifting from reactive to proactive and predictive care. Retrieved from https://www.marsdd.com/newsand-insights/transforming-health-shifting-from-reactive-toproactive-and-predictive-care/

Sadana, R., Blas, E., Budhwani, S., Koller, T., \& Paraje, G. (2016). Healthy ageing: Raising awareness of inequalities, determinants, and what could be done to improve health equity. The Gerontologist, 56(Suppl. 2), S178-S193. doi:10.1093/geront/gnw034

Salvi, F., Morichi, V., Grilli, A., Giorgi, R., De Tommaso, G., \& DessìFulgheri, P. (2007). The elderly in the emergency department: A critical review of problems and solutions. Internal and Emergency Medicine, 2(4), 292-301. doi:10.1007/s11739-007-0081-3

Samaras, N, Chevalley, T., Samaras, D., \& Gold, G. (2010). Older patients in the emergency department: A review. Ann Emerg Med, 56(3), 261-269. doi:10.1016/j.annemergmed.2010.04.015 


\section{Appendix A: Literature search}

\section{Table 1: Medline Literature Search}

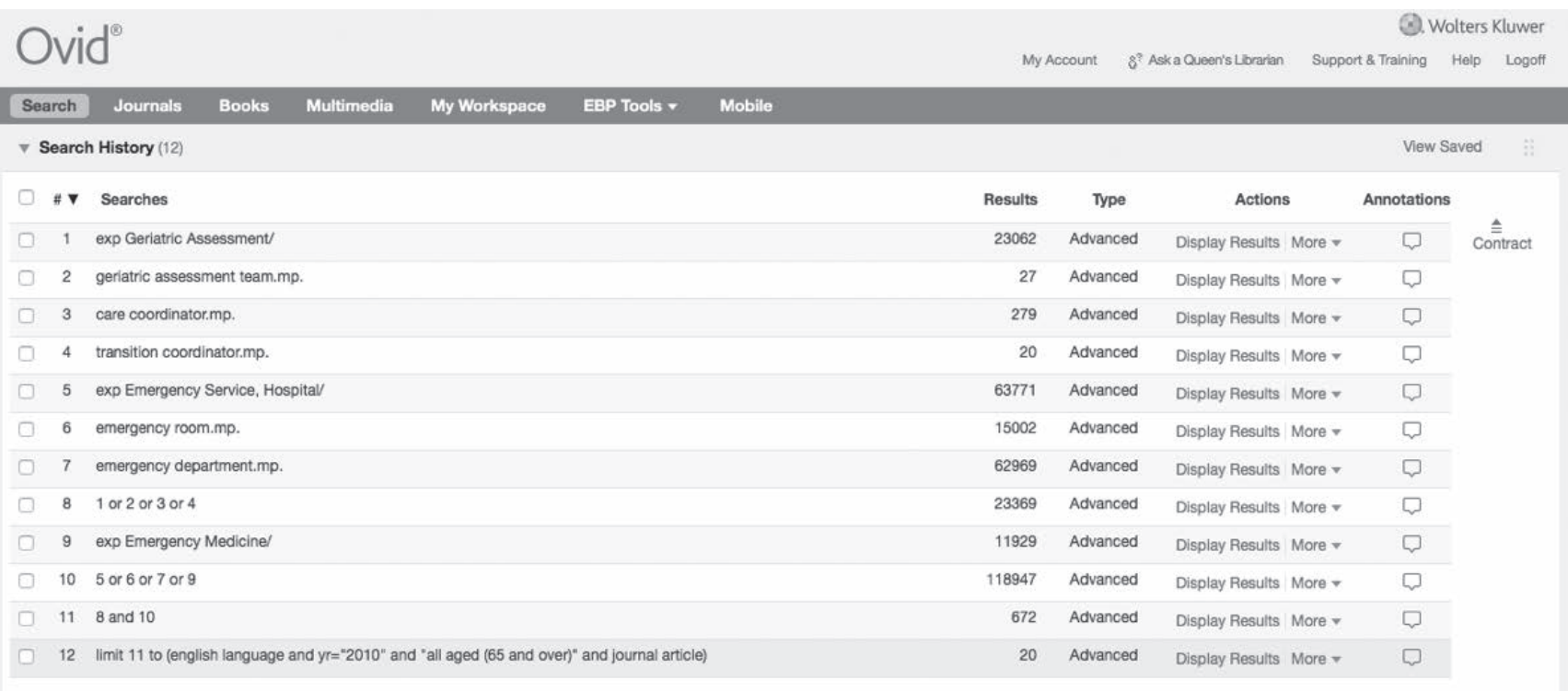

\section{Table 2: CINAHL Literature Search}

\begin{tabular}{|c|c|c|c|c|c|c|}
\hline & Search & Search Terms & Search Options & \multicolumn{3}{|l|}{ Actions } \\
\hline$\square$ & s7 & S1 AND S2 & $\begin{array}{l}\text { Limiters - Linked Full Text } \\
\text { Expanders - Apply related words } \\
\text { Narrow by SubjectGeographic: - usa } \\
\text { Narrow by SubjectAge: - aged: } 65+\text { years } \\
\text { Narrow by Language: - english } \\
\text { Search modes - Boolean/Phrase }\end{array}$ & Q View Results (25) & (i) View Detalls & $\square$ Edit \\
\hline D & s6 & ه S1 AND S2 & $\begin{array}{l}\text { Limiters - Linked Full Text } \\
\text { Expanders - Apply related words } \\
\text { Narrow by SubjectAge: - aged: } 65+\text { years } \\
\text { Narrow by Language: - english } \\
\text { Search modes - Boolean/Phrase }\end{array}$ & a) View Results (37) & (i) View Details & $\square$ Edit \\
\hline D & S5 & \& $\mathrm{S} 1$ AND $\mathrm{S} 2$ & $\begin{array}{l}\text { Limiters - Linked Full Text } \\
\text { Expanders - Apply related words } \\
\text { Narrow by Language: - english } \\
\text { Search modes - Boolean/Phrase }\end{array}$ & (a) View Results (43) & (i) Vlew Detalls & $\triangle$ Edit \\
\hline D & S4 & ه $\mathrm{S} 1$ AND $\mathrm{S} 2$ & $\begin{array}{l}\text { Limiters - Linked Full Text } \\
\text { Expanders - Apply related words } \\
\text { Search modes - Boolean/Phrase }\end{array}$ & Q Vlew Results (43) & (i) Vlew Detalls & $\triangle$ Edit \\
\hline 0 & s3 & ه $\mathrm{S} 1$ AND S2 & $\begin{array}{l}\text { Expanders - Apply related words } \\
\text { Search modes - Boolean/Phrase }\end{array}$ & a View Results (228) & (i) Vlew Detalls & $\triangle$ Edit \\
\hline$\square$ & s2 & $\begin{array}{l}\text { (MH "Emergency Service") OR "emergency department" OR } \\
\text { "emergency room" OR (MH "Emergency Medicine") }\end{array}$ & $\begin{array}{l}\text { Expanders - Apply related words } \\
\text { Search modes - Boolean/Phrase }\end{array}$ & a) Vlew Results $(48,146)$ & (i) View Details & Is $\square$ Edit \\
\hline$\square$ & s1 & $\begin{array}{l}\text { (MH "Geriatric Assessment) OR "geriatric assessment team" OR } \\
\text { (MH "Nursing Care Coordination (Saba CCC)") OR "care coordinator" } \\
\text { OR "transition coordinator" }\end{array}$ & $\begin{array}{l}\text { Expanders - Apply related words } \\
\text { Search modes - Boolean/Phrase }\end{array}$ & Q Vlew Resuits $(7,213)$ & (i) View Details & $\square$ Edit \\
\hline
\end{tabular}

